\title{
Successful Treatment of Listeria Meningitis in a Pregnant Woman with Ulcerative Colitis Receiving Infliximab
}

\author{
Lamdhade S $\mathbf{J}^{1}$, Thussu $\mathbf{A}^{1}, \mathbf{A}$ Benwan $\mathbf{K O}^{2}$ and Alroughani $\mathbf{R}^{1 *}$ \\ ${ }^{1}$ Division of Neurology, Department of Medicine, Amiri Hospital, Kuwait \\ ${ }^{2}$ Department of Microbiology, Amiri Hospital, Kuwait
}

\begin{abstract}
Objectives: To report a case of Listeria monocytogen meningitis; a rare complication during Infliximab therapy for ulcerative colitis during early pregnancy.

Case presentation and intervention: A 28 year old woman was treated with prior immunosuppression and recent infliximab for ulcerative colitis. Pregnancy was confirmed at second infliximab infusion. Five days after the third dose, she developed signs of acute meningitis with subsequent VI cranial neuropathy. Cerebrospinal fluid Gram-stain suspected listeria monocytogen organisms, which was confirmed by blood and cerebrospinal fluid cultures. Meningitis was successfully treated with Ampicillin and Gentamycin. Spontaneous Intrauterine death of fetus occurred at 15 weeks gestation.
\end{abstract}

Conclusions: This case highlights the importance of high index of suspicion of opportunistic infections such as Listeria meningitis with the use of infliximab.

\section{Introduction}

Infliximab (Remicade) is a chimeric IgG1 monoclonal antibody and has a high specificity and affinity to Tumor necrosis factor alpha (TNF- $\alpha$ ). It is frequently used as disease modifying agent in refractory cases of Inflammatory Bowel Disease (IBD) and Rheumatoid Arthritis (RA) [1,2]. However emergence of opportunistic infections such as mycobacteria, listeria monocytosis, nocardiosis, and invasive aspergilosis raised safety concerns.

Listeria monocytogen is gram-positive bacilli commonly isolated from environmental sources (water and food such as cheese, milk products, and undercooked meat). It can cause sporadic or epidemic infections and can be found in feces of $1-5 \%$ asymptomatic healthy adults. Immunosuppression, defective cell mediated immunity and pregnancies are considered high-risk conditions.Case mortality with central nervous system (CNS) infection is very high reaching $27 \%$ and many patients are left with neurological sequel [3]. Though listeria meningitis has been described with the use of infliximab, its occurrence during pregnancy in ulcerative colitis is rare. We report a case of listeria meningitis in a woman who received infliximab during pregnancy.

\section{Case Report}

A 28-year-old married female was diagnosed with ulcerative colitis in May 2006. She was given Mesalamine and Azathioprine was subsequently added in 2008 as a disease modifying Therapy. However, both were stopped for seven months since she was planning to get pregnant. The symptoms of ulcerative colitis reappeared. Hence Mesalamine, azathioprine and oral prednisolone $40 \mathrm{mg}$ daily were instituted in December 2009. Infliximab was started due to increased disease activity manifested by frequent bloody diarrhea in February 2010. Prior to her second infliximab infusion, she noticed amenorrhea and pregnancy was confirmed by abdominal ultrasound. She continued to receive infliximab, as there was no absolute contraindication for its use during pregnancy. On $5^{\text {th }}$ April 2012, she received the third infliximab infusion. Five days later (11 weeks gestation), she presented with body ache, fever and severe headache for four days. She looked sick, toxic and had fever of $39.3^{\circ} \mathrm{C}$. The sequence of events occurred in the case was summarized in Figure 1. Mild diffuse abdominal tenderness was noted. Except for neck stiffness, her systemic and neurological examinations were unremarkable. Her complete blood count showed WBC $11.4 \times 10^{9}$,

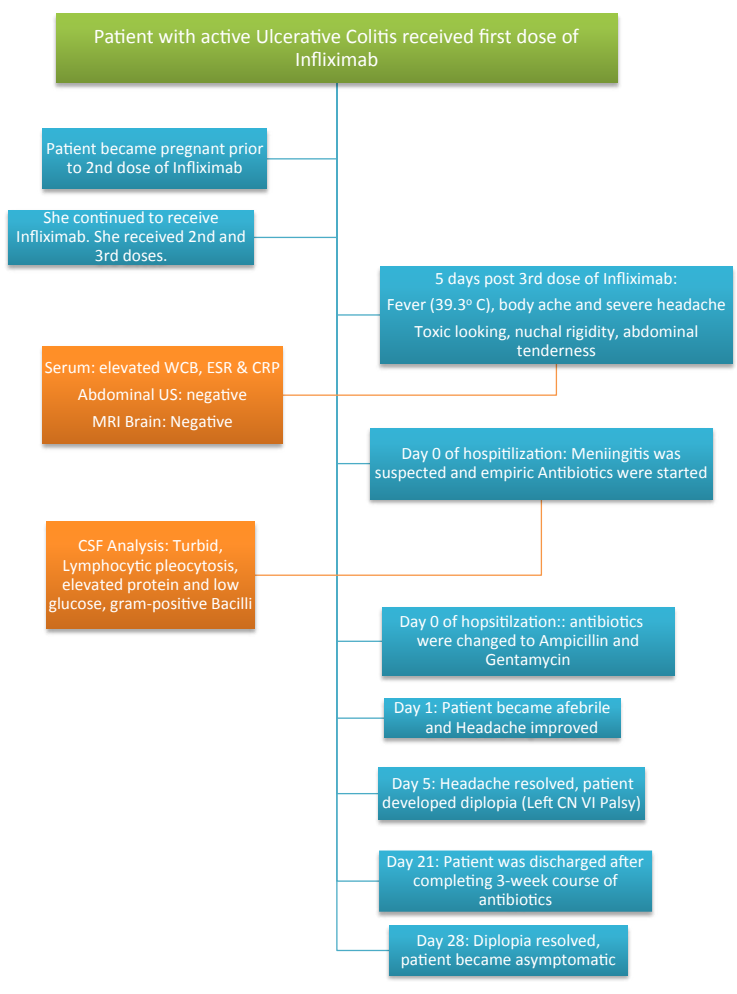

Figure 1: Sequence of events observed in the patient.

*Corresponding author: Raed Alroughani, Division of Neurology, Department of Medicine, Amiri Hospital, PO BOX. 1661, Qurtoba, 73767, Kuwait, Tel: +965 22450005, Fax: +965 22467499; E-mail: alroughani@gmail.com

Received August 26, 2013; Accepted September 06, 2013; Published September 12, 2013

Citation: Lamdhade SJ, Thussu A, Al Benwan KO, Alroughani R1 (2013) Successful Treatment of Listeria Meningitis in a Pregnant Woman with Ulcerative Colitis Receiving Infliximab. Gen Med (Los Angel) 1: 116. doi: 10.4172/23275146.1000116

Copyright: ( 2013 Lamdhade SJ, et al. This is an open-access article distributed under the terms of the Creative Commons Attribution License, which permits unrestricted use, distribution, and reproduction in any medium, provided the original author and source are credited. 